\title{
Assessment of a Microbicide Candidate among a Diverse Cohort of Urban Southern US Women and their Male Sexual Partners
}

Paula Frew ${ }^{1,2,3,4^{*}}$, Kimberly Parker ${ }^{5}$, Takeia Horton ${ }^{3,4}$, Brooke Hixson ${ }^{1,3,4}$, Lisa Flowers ${ }^{6}$, Frances Priddy ${ }^{1,7}$, Lisa Grohskopf ${ }^{8}$, Christine Mauck ${ }^{9}$ and Kimberly Workowski ${ }^{1,2,3}$

${ }^{1}$ Emory University School of Medicine, Department of Medicine, Division of Infectious Diseases, USA

${ }^{2}$ Emory Center for AIDS Research, USA

${ }^{3}$ The Hope Clinic of the Emory Vaccine Center, USA

${ }^{4}$ Emory University, Rollins School of Public Health, USA

${ }^{5}$ Texas Woman's University, USA

${ }^{6}$ Emory University School of Medicine, Department of Obstetrics and Gynecology, USA

${ }^{7}$ International AIDS Vaccine Initiative, USA

${ }^{8}$ United States Centers for Disease Control and Prevention, USA

${ }^{9}$ CONRAD, USA

\begin{abstract}
Background: This mixed methods study reports on product acceptance from a Phase I clinical trial of a candidate non-nucleoside reverse transcriptase inhibitor (NNRTI) vaginal microbicide product (UC781). The product was evaluated in the context of a Phase I clinical trial in an area characterized by high HIV prevalence among minority women. The findings will inform the development of an acceptable microbicide that will address the needs of diverse women and their partners.

Methods: This is a mixed methods study of 34 racially and ethnically diverse female participants and 10 male partners in Atlanta, Georgia. Chi-square tests for marginal homogeneity and kappa statistics were calculated to analyze differences between groups on product attributes and use intention. ANOVA was used to examine difference between the treatment groups. Qualitative data were analyzed via constant comparative methodology.

Results: Thirty-four out of the original female cohort of 36 completed the questionnaire. Approval of future microbicide development was high at $91.2 \%(n=31)$ despite a lack of enthusiasm for the placebo and UC781 formulations. Overall female acceptability was correlated with personal protection motivation $(r=1.00, p<0.001)$. African American women indicated greater likelihood of post-licensure microbicide use $\left(X^{2}{ }_{(3)}=7.9, p=0.048\right)$ and ascribed greater importance to its potential protection against $\operatorname{HIV}\left(X^{2}=18.7, p=0.001\right)$ and its potential for dual protection (protective against STIs and/or pregnancy) compared to white women $\left(X_{(4)}^{2}=11.3, p=0.024\right)$. Men and women supported development in the form of an intravaginal ring or suppository. Men were more likely to encourage female adoption of the method if it afforded HIV protection $(r=0.935, p=0.001)$.

Conclusions: Although most women agreed that the development of a microbicide was an important endeavor quantitative and qualitative data indicated they would not use placebo or UC781 due to the objectionable viscosity, odor, and color. Male partners felt the potential protective benefit of a future microbicide product was its most important feature.
\end{abstract}

Keywords: HIV/AIDS; Microbicide; Women; Minorities; Clinical trials; Product acceptability

\section{Introduction}

In the United States, the number and proportion of HIV/AIDS cases among women, continue to highlight the need for new and effective prevention strategies against HIV and other sexually transmitted infections (STIs) [1,2]. Females in all racial and ethnic categories have been affected, particularly black/African American women living in the South $[1,3]$. Inequitable decision-making and compromised power have been identified as primary obstacles to women's ability to negotiate condom use [4-7]. Microbicides may therefore offer women an important protective option.

Products previously tested in clinical trials demonstrated mixed efficacy results, including the most recent positive development $[8,9]$ with a $1 \%$ vaginal gel formulation of tenofovir, which reduced HIV acquisition in South African women by $39 \%$ overall and by $54 \%$ among those with high gel adherence $[10,11]$. UC781 is a non-nucleoside reverse transcriptase inhibitor (NNRTI) of the HIV-1 reverse transcriptase (RT) enzyme which has the potential to act against a wide range of HIV-1 isolates, including laboratory adapted strains and primary isolates of all major clades (A through $\mathrm{G}$ ). Following in vitro and ex vivo preclinical toxicity studies of UC781 gel formulations at $0.1 \%$ and $1 \%$, the UC781 intravaginally administered microbicide proceeded to Phase-I clinical testing with 48 sexually abstinent women in the United States in 2003 [12].

Because microbicide safety and tolerability is best evaluated in a context which closely reflects conditions of actual use, we conducted a Phase-I UC781 trial with 36 sexually active women and their male

*Corresponding author: Paula M. Frew, Emory University, Department of Medicine, Division of Infectious Diseases, The Hope Clinic of the Emory Vaccine Center, 603 Church Street, Decatur, GA 30030, USA, Tel: 404-712-8546; Fax: 404712-9017; E-mail: pfrew@emory.edu

Received May 07, 2012; Accepted June 28, 2012; Published July 03, 2012

Citation: Frew P, Parker K, Horton T, Hixson B, Flowers L, et al. (2012) Assessment of a Microbicide Candidate among a Diverse Cohort of Urban Southern US Women and their Male Sexual Partners. J AIDS Clinic Res S4:004. doi:10.4172/2155-6113.S4-004

Copyright: () 2012 Frew P, et al. This is an open-access article distributed under the terms of the Creative Commons Attribution License, which permits unrestricted use, distribution, and reproduction in any medium, provided the original author and source are credited. 
partners. Our objective was to assess the acceptability of $0.1 \%$ and $0.25 \%$ UC781 gel use by female participants and their male partners. The $0.1 \%$ and $0.25 \%$ UC781 gel was a light yellow color while the placebo gel was clear. Both were administered in plastic, pre-filled applicators. The applicators were identical to preserve blinding among study personnel and participants. Participants were not aware of the color distinction in the gels.

This study focused on Southern women enrolled in one of the first Phase-I microbicide clinical trials in this region. This study offers unique insight into dynamic intersecting factors potentially influential in future product adoption among priority populations.

\section{Methods}

\section{Study sample}

Data were gathered from a larger clinical trial. The single center, double-blind, randomized, staged, placebo-controlled "Phase I Study of the Safety and Acceptability of UC781 Topical Vaginal Microbicide in Heterosexual Women and their Male Partners" was conducted from 2007 to 2008 and was sponsored by CONRAD (Arlington, Virginia) with funding from the Centers for Diseases Control and Prevention in Atlanta, Georgia. Sexually active HIV-uninfected women were randomized in a 1:1:1 ratio to apply $0.1 \%$ UC781 Gel, $0.25 \%$ UC781 Gel or placebo gel intravaginally twice-daily for 14 days. The placebo gel is the so-called "Universal Placebo," designed for use in microbicide trials and felt unlikely to affect safety endpoints and HIV acquisition [13].

Eligible females were age 18-45, HIV-negative, not pregnant or planning to become pregnant within six months, having regular menstrual cycles, and in a mutually monogamous sexually active relationship with one male partner. Women agreed to inform their male partner about their participation in the study and couples agreed to use study-provided male latex condoms for each act of vaginal intercourse for the duration of the study. Study procedures included insertion of one dose $(3.5 \mathrm{ml})$ of gel via applicator twice daily at a minimum of 8-hour dose increments (and up to one hour before coitus) for two weeks for evaluation of the product, daily study diary entries, and five study visits inclusive of participation in a focus group or interview. Clinical procedures included physical examinations, HIV and STI counseling and testing, $\mathrm{CBC}$, chemistry, urinalysis, Pap smear, testing for Chlamydia, gonorrhea, syphilis and HIV, vaginal microflora culture, and colposcopic examination and digital image collection.

Women were recruited at women's health centers, college campuses, and churches during 2007 - 2008. Written informed consent was obtained from all participants. Compensation for women's time and travel costs was provided for the five study visits (\$50 per visit). Recruitment of male partners occurred through female participants. Men that were willing to participate in the study were invited to the study site and consented to participate in the questionnaire component of the study. Those who declined to complete a survey were therefore not included in the study. Male participants were compensated $\$ 25$ for completion of the questionnaire. Emory University and the Centers for Disease Control and Prevention institutional review boards approved the study.

\section{Data collection}

This study employed a mixed methods approach to understand the participants' comparative experiences with UC781 and placebo. Women completed a 37 -item questionnaire at their final visit. Male partner data were collected via a 19-item survey at the day-14 visit.
All female study volunteers were asked to participate in the qualitative component. Because of the sensitive nature of the qualitative inquiry, women who refused to participate in focus groups were offered an alternative of a semi-structured interview. Topic guides for focus groups and interviews were developed with a team of clinicians, behavioral researchers, and community members to ensure content face validity. Particular care was taken in crafting scripts that facilitated a conversation in the vernacular of the participants, but was detailed in its potential probes for all questions. Standardized questions elicited information about the product's physical properties, product effect on sexual experiences, and ideal attributes of a microbicide.

Focus groups were typically comprised of 4 to 8 group members. Focus groups and interviews were conducted by a trained African American woman with experience working with similar study populations. All responses were audio recorded. The interviews and focus groups were conducted in private rooms at the clinic. During the course of the focus groups and interviews, participants' responses were routinely read back to them by the interviewer to ensure correct interpretation of responses (member-checking process). Interviews were audiotaped and notes were taken. The tapes were later transcribed by a member of our research team.

\section{Quantitative data analysis}

Quantitative data were analyzed via SAS (Cary, NC). Descriptive statistics, bivariate analysis, and one-way ANOVAs were performed. Differences between inter-partner attitudes towards gel use were analyzed with chi-square tests for marginal homogeneity and kappa statistics. The Levene test was performed to test for homogeneity of variances between the groups. Overall acceptability was defined as the proportion of female and male participants who were "somewhat likely" or "very likely" to use $0.1 \%$ and $0.25 \%$ UC781 gel during sexual intercourse in the future.

Reliability of scaled response items was also assessed. The two sexual pleasure scales were constructed to examine the product experience in the context of sexual intercourse and its perceived favorability to partners. The personal protection scale measured the perceived importance of the product in protecting against HIV, STIs, and pregnancy. We assessed women's overall acceptability by measuring her comfort with the product, problems with usage, likelihood of recommending the product, and potential adoption via the "Product Adoption Scale", which was comprised of questions regarding comfort with using the gel, problems with the gel, willingness to recommend, and acceptability of the gel. Male acceptability was assessed by a singleitem: "If the gel was approved by the FDA to lower the risk of spreading HIV through sex, would you prefer that your partner use it?"

\section{Code sheet development and qualitative procedures}

A detailed code sheet and coding scheme were developed to capture relevant details from the transcripts such as attitudes, beliefs, partner experiences, and social/cultural dimensions that may influence microbicide use. The 8 thematic categories were developed by independent content review of all transcripts, followed by discussion among the research team about emergent themes. A consensus on comparison constancy was reached by three persons conducting the data analysis [14].

Analyses utilized the constant comparative approach within the grounded theory process model, which employs both deductive and inductive methods to identify patterns or themes [15]. Codes were 


\begin{tabular}{|l|c|}
\hline & $\mathbf{n = 3 4}(\%)^{*}$ \\
\hline Race & \\
Black/African American & $15(44.1 \%)$ \\
Caucasian & $18(52.9 \%)$ \\
Multiracial & $1(2.9 \%)$ \\
\hline Educational Attainment & \\
High School Graduate & $2(5.6 \%)$ \\
Some college, no degree & $6(17.6 \%)$ \\
Associate/Vocational & $2(5.6 \%)$ \\
Bachelor & $12(35.3 \%)$ \\
Masters/Professional & $8(22.22 \%)$ \\
Doctorate & $2(5.6 \%)$ \\
Missing & $2(5.6 \%)$ \\
\hline Motivation to Participate & \\
Altruism & $8(23.5 \%)$ \\
Compensation & $10(29.4 \%)$ \\
Personal connection to the cause & $5(14.7 \%)$ \\
Scientific medical contribution & $8(23.5 \%)$ \\
Other & $3(8.8 \%)$ \\
\hline Median Age (IQR) & $31(19-43)$ \\
\hline
\end{tabular}

*Thirty six women enrolled in the study but two women did not complete the demographic instrument

Table 1: Demographic Characteristics of Women Enrolled in a Microbicide Clinical Trial in Atlanta, GA (2007/2008).

refined in a series of iterative cycles using methods developed by investigators at the Centers for Disease Control and Prevention (CDC) for team-based qualitative analyses [16]. Intercoder reliability was $93.3 \%$, a value higher than the recommended $90 \%$ agreement level for qualitative data analysis.

\section{Results}

\section{Participant characteristics}

Thirty-six women were enrolled in the study in 2007-2008. Of these, 34 completed the survey and participated in the focus groups or interviews. In addition, ten male partners completed the survey. The median age of the women who participated in the study was 31 years (range: 19 to 43 years). Ethnicity was nearly evenly distributed with black/African Americans $(\mathrm{N}=15,44.1 \%)$ and Caucasians $(\mathrm{N}=18$, $52.9 \%)$ comprising the majority, and one woman self-identified as multiracial $(\mathrm{N}=1,2.9 \%)$ (Table 1$)$.

\section{Scale measures}

The four scales - "Decrease in Sexual Pleasure Scale," "Increase in Sexual Pleasure Scale," "Personal Protection Scale," and "Product Adoption Scale" - all achieved satisfactory internal consistency $(\alpha=0.652-0.757)$. The male questionnaire three scales - "Partner Protection Scale," "Male Sexual Pleasure Scale," and "Male Product Properties Scale" - also had high internal consistencies $(\alpha=0.706-$ 0.956).

\section{Product experience}

Female use: Ninety-two percent of the female participants $(\mathrm{N}=31)$ were comfortable using the gel, but were not enthusiastic about its current form. Of those women who responded on gel use if it were approved, 30\% $(\mathrm{N}=6)$ said they would be very likely to use it, $40 \%$ $(\mathrm{N}=8)$ said they would be somewhat likely to use it, and $30 \%(\mathrm{~N}=6)$ said they would be unlikely to use it. Women's responses to the gel differed significantly by ethnicity. Black/African American women were more likely to use the gel if it were available. Of the women who answered the future use question, all black/African American women $(\mathrm{N}=9)$ said they would be either somewhat or very likely to use the gel, whereas $60 \%$ of Caucasian women $(\mathrm{N}=6)$ said they would be unlikely to use it $\left(\chi^{2}=7.9, \mathrm{p}=.048\right)$. When asked how important HIV protection alone was, the majority of African American women $(\mathrm{N}=13,87 \%)$ indicated that it was very important compared to only $17 \%(\mathrm{~N}=3)$ of Caucasian women $\left(\chi^{2}=18.7, p=0.001\right)$. Furthermore, when women wrote in what they liked about the product, $80 \%(\mathrm{~N}=12)$ of African American women said they valued that the gel might offer "added protection" against HIV. In contrast, only $50 \%(\mathrm{~N}=9)$ of Caucasian women wrote they most valued its potential HIV protection $\left(\chi^{2}=4.78, \mathrm{p}=0.092\right)$.

The importance of the microbicide functioning as a dual protection barrier also differed by ethnicity $\left(\chi^{2}=11.3, \mathrm{p}=0.024\right)$. Black/African American women were in strong favor of a dual protection gel that protected against HIV, STIs, and pregnancy $(\mathrm{N}=13,87 \%)$, while only $44 \%(\mathrm{~N}=8)$ of white women felt a dual protection gel was very important. This group was also more interested in a gel that protected against HIV (black/African Americans: $\mathrm{N}=13,87 \%$, whites: $\mathrm{N}=3,17 \%$ ) or against pregnancy (black/African Americans: $\mathrm{N}=8,53 \%$, whites: $\mathrm{N}=7,39 \%)$.

Most women stated in interviews and in focus groups they would use the microbicide gel if it was offered in another form such as a vaginal ring. Participants familiar with the contraceptive vaginal ring described it as "awesome" and "something you don't have to worry about." Other suggestions included a "non-hormonal product," a product that did not seem "wasteful," an inexpensive product, or a "patch."

There were no significant differences between the two treatment groups and the placebo group with regards to most the gel's characteristics (odor, irritation, color, staining), effect on sexual pleasure, importance of protection, or likelihood of future use. With respect to the product's physical attributes, most women $(\mathrm{N}=22,64.7 \%)$ had neutral responses to the gel's color (regardless of treatment group) with $8.8 \%(\mathrm{~N}=3)$ expressing disfavor and $8.8 \%(\mathrm{~N}=3)$ indicating some acceptance for it. A small proportion of the group $(\mathrm{N}=6,17.6 \%)$ liked its color. Those who had the placebo gel described it as "totally clear," "like water," and having "no color at all." The treatment gel was referred to as an "odd yellow" and "lemon-y" color. Participants in both placebo and treatment groups showed a preference for a clear gel with $73 \%(\mathrm{~N}=25)$ of the overall cohort agreeing a clear gel would be preferable.

Fifty-five percent $(\mathrm{N}=20)$ of participants felt the gel was too messy (viscosity). Women who received the placebo felt it was messier than women who received either the $0.1 \%$ or the $0.25 \%$ formulation $(\mathrm{F}=3.79, \mathrm{p}=0.034)$.The products were described as "watery, "messy," and "thin and "runny." Many of the women that experienced "leakage" reported they wore panty liners for all or a majority of the time while participating in the study. In addition, women varied on whether the gel stained underwear with most indicating strong disagreement $(\mathrm{N}=22$, $64.7 \%)$ and others expressing agreement $(\mathrm{N}=12,35.3 \%)$ (Table 2).

The gel scent was an issue with $32.4 \%(\mathrm{~N}=11)$ of the women reporting an odor. Most described the odor as unpleasant. One of the participants indicated the gel released odor based on interaction with her body temperature. Another woman stated she did not notice a scent until the gel was discharged from her body.

Participant statements were also mixed on any gel-related physical sensations. Only $11.8 \%(n=4)$ said they had explicit problems with the product. Women commented that the products resulted in "mild discomfort" and offered that they were "uncomfortable" due to "tingling," "itching," and "burning" sensations.

Participants generally did not have problems using the gel applicator. Respondents liked the applicator with unanimous agreement on its ease 


\begin{tabular}{|c|c|c|c|c|c|}
\hline \multirow[t]{2}{*}{ Factor } & \multicolumn{3}{|c|}{ Treatment Group } & \multirow[t]{2}{*}{$\mathbf{F}$} & \multirow[t]{2}{*}{$\mathrm{p}$-value } \\
\hline & $\begin{array}{c}0.1 \% \\
(n=12)\end{array}$ & $\begin{array}{l}0.25 \% \\
(n=10)\end{array}$ & $\begin{array}{c}\text { Placebo } \\
(\mathrm{n}=12)\end{array}$ & & \\
\hline Product acceptability & & & & 1.54 & 0.24 \\
\hline Very Likely to use & 4 & 0 & 2 & & \\
\hline Likely to use & 2 & 5 & 1 & & \\
\hline Unlikely to use & 1 & 0 & 5 & & \\
\hline Missing & 5 & 7 & 4 & & \\
\hline Experienced Irritation & & & & 0.40 & 0.67 \\
\hline Yes- Experienced Irritation & 3 & 1 & 2 & & \\
\hline No - Did Not Experience Irritation & 9 & 9 & 10 & & \\
\hline Experienced Odor & & & & 0.26 & 0.31 \\
\hline Yes-Experienced Odor & 3 & 4 & 4 & & \\
\hline No - Did Not Experience Odor & 9 & 6 & 8 & & \\
\hline Reported Unpleasant Odor & & & & 0.05 & 0.95 \\
\hline Yes - Reported Unpleasant Odor & 2 & 2 & 3 & & \\
\hline $\begin{array}{c}\text { No-Did Not Report Unpleasant } \\
\text { Odor }\end{array}$ & 10 & 8 & 9 & & \\
\hline Would prefer a clear gel & & & & 0.67 & 0.52 \\
\hline Agree & 10 & 8 & 7 & & \\
\hline Neutral & 2 & 2 & 5 & & \\
\hline Viscosity & & & & 3.79 & 0.03 \\
\hline Too messy & 5 & 6 & 9 & & \\
\hline Not too messy & 6 & 3 & 0 & & \\
\hline $\begin{array}{l}\text { Willingness to recommend } \\
\text { product to others }\end{array}$ & & & & 0.56 & 0.58 \\
\hline Yes & 12 & 9 & 11 & & \\
\hline No & 0 & 1 & 1 & & \\
\hline Male partner acceptability & & & & 0.21 & 0.81 \\
\hline Likely to use & 2 & 1 & 0 & & \\
\hline Neutral & 0 & 1 & 0 & & \\
\hline Unlikely to use & 1 & 2 & 1 & & \\
\hline Missing & 9 & 6 & 11 & & \\
\hline
\end{tabular}

Table 2: Product Acceptability Differences between Treatment Groups, Atlanta, Georgia (2007/2008).

of use $(\mathrm{N}=34,100 \%)$ and ease of insertion $(\mathrm{N}=25,73.5 \%)$. A smaller proportion $(\mathrm{N}=8,23.5 \%)$ indicated some favor for it, and one woman was neutral (2.9\%).

The majority, $94.1 \%(\mathrm{~N}=32)$, of women said they would recommend the gel to other women if it were available. Common reasons included ease of use, a lack of side effects, independent protection decisionmaking, and protection against HIV. Recommended changes included improving the gel's physical properties by manufacturing a clear, thick, and odorless gel.

Female and male acceptability: Women's perceived need for personal protection was robustly correlated with product acceptability $(\mathrm{r}=0.522, \mathrm{p}=0.018)$. Men were motivated by the product's potential to protect their partners against HIV alone, or in combination against STIs and pregnancy $(\mathrm{r}=0.980, \mathrm{p}<0.001)$. There were no significant differences between the placebo and treatment groups with regard to product acceptability $(\mathrm{F}=1.54, \mathrm{p}=0.24)$, willingness to recommend the product $(\mathrm{F}=0.56, \mathrm{p}=.58)$, or male partner product acceptability $(\mathrm{F}=0.21$, $\mathrm{p}=0.81)$.

Partner/Sexual experiences with product: While $88.2 \%(\mathrm{~N}=30)$ of participants noticed the gel immediately after the insertion of the product, only $35.3 \%(\mathrm{~N}=12)$ noticed it during sex. Women who felt the gel decreased their sexual pleasure were less likely to use the gel $(\mathrm{r}=-0.487, \mathrm{p}=0.030)$. Concordance within couples was mixed. Couples agreed most that they noticed the gel during sex (kappa $=0.550)$ and that the gel added to male sexual pleasure (kappa=.557). One participant stated "the gel just made the condoms more fun" and her partner enjoyed the sensation caused by the gel. Moderate agreement was found on the gel's odor (kappa $=0.426)$ and the likelihood of future use (kappa $=0.444)$. Couples weakly agreed on gel characteristics such as consistency (kappa $=0.328$ ) and color (kappa $=0.308)$ as well as the gel's effect on female sexual pleasure $(\mathrm{k}=0.143)$. Male partners had mixed reactions to the gel's effect on their sexual pleasure. Slightly more than half of the male partners who were sampled believed the gel decreased their sexual pleasure $(55.6 \%, \mathrm{~N}=5)$, but $33 \%(\mathrm{~N}=3)$ of the men felt the gel increased sexual pleasure.

Although some of the women felt as if the gel could be used as a lubricant, sexual intercourse with the gel was described as "messy," a characteristic some of the women did not like because "[the gel] got everywhere so that was not good." One participant was concerned about her partner's reaction to the gel:

I didn't like, let him put his hands down there at all. I was very selfconscious about it...I was a little nervous about it being greasy so that was a little different...

Participants also felt the gel hindered sexual experience because intercourse "had to be scheduled" and "limited spontaneity." This was generally viewed as an inconvenience. One participant stated it was difficult for her to predict if and when she and her partner would engage in sexual intercourse. Another participant described the gel insertion time as "awkward" because the partners would have to plan sexual intercourse around the gel insertion time, something she did not enjoy. The gel also limited certain acts the women and their partners engaged in such as oral sex. One participant stated "as far as sexually, you couldn't have oral sex so that was a turn off." However, one woman stated:

That's what it was [enhanced intimacy]. The very first time we engaged in sex, he was more like thinking I was really, really ready, you know like the foreplay was great, and I was like no, I just used the gel. But...he thought it was really great because I was more lubricated than normal.

Almost all, 91.2\% ( $\mathrm{N}=31)$, women said if they needed HIV protection they would use a similar product. Common reasons given for using the gel were potential HIV protection $(61.8 \%, \mathrm{~N}=21)$, ease of use $(38.2 \%, \mathrm{~N}=13)$, discretion $(11.8 \%, \mathrm{~N}=4)$ and precoital application. Women stated that it would enhance their opportunity to negotiate with partners and adopt safer sex practices. A participant commented:

"When I think about when I was an undergrad and among my peers, if you had a condom in your purse, you had to negotiate that, or if you were with a person that you were not in a relationship with, then that could be a mess. You know, an emotional mess, so if you stick [the gel/applicator] in your purse, and go to the bathroom, and you can have sex with that person and be protected from STDs and pregnancy, then yeah, I would use it" (Table 3 ).

\section{Discussion}

Among this group we found a majority (70\%) of our trial participants would use a microbicide product in the future [17-21]. There were no significant differences between the placebo and treatment groups with regard to acceptability and product characteristics, with 


\begin{tabular}{|c|c|}
\hline Leakage & $\begin{array}{l}\text { The gel itself, it seems like I would put it in and it would leak back out and then .....most of it would leak back out and rest would leak out } \\
\text { very slowly or I don't know if my body was trying to flush it our or what but I had a lot of leakage. } \\
\text { Even on day } 10 \text { when I was really frustrated with it, and then I was thinking if this was my only way to prevent HIV, "would I do it anyway?" } \\
\text { If they had to use [a panty liner] every day...if...you only had to wear one after you applied [the gel], or after you had sex but I could see } \\
\text { a problem with having to wear one every day. }\end{array}$ \\
\hline Scent & I hated [the scent]. I could smell it even during the day and I didn't like it so I would change the scent. \\
\hline Applicator & $\begin{array}{l}\text { I thought that maybe [gel] was going to fill up the [applicator] and... and I couldn't figure that out. } \\
\text { I was not sure if [the applicator] was in all the way. It did not seem that I was using it as some type of protection and it didn't seem that it } \\
\text { was up against the cervix or that the gel was reaching the cervix. }\end{array}$ \\
\hline $\begin{array}{c}\text { Sexual } \\
\text { Enhancement }\end{array}$ & $\begin{array}{l}\text { My partner and I...use condoms because I didn't want [anymore] more children. So the gel just made the condoms more fun. [My } \\
\text { partner] said he felt like he had more sensation with the gel. I think if it is promoted, if it's advertised as almost a sexual enhancement, I } \\
\text { think it would probably be more wide spread }\end{array}$ \\
\hline Sexual Limitations & $\begin{array}{l}\text { As far as sexually, you couldn't have oral sex so that was a turn off. } \\
\text {...no using your hands, no mouth, nothing thing. Like for us it was very serious issue because generally I favor those things }\end{array}$ \\
\hline Insertion Timing & $\begin{array}{l}\text { Timing issues could be a little awkward especially if sex is not plan } \\
\text { [The timing] wasn't that bad, especially since it was only for a couple of weeks. So that was okay. But if it had to be everyday, all the time, then I } \\
\text { would have a problem with it. }\end{array}$ \\
\hline $\begin{array}{l}\text { Difficulty with } \\
\text { Study Regimen }\end{array}$ & $\begin{array}{l}\text {...if [the gel] had to be [inserted] everyday, all the time, then I would have a problem with it. } \\
\text {...it depended on the conditions that you were using [the gel]...but someone like me, [inserting the gel] was a chore. }\end{array}$ \\
\hline Partner Response & $\begin{array}{l}\text {...in the beginning. But when I explained it to him like Jane explained it to me, he was okay with it when I gave him more understanding } \\
\text { of what was the purpose and all that. He just wanted to know more about it. }\end{array}$ \\
\hline
\end{tabular}

Table 3: Qualitative Themes and Quotations.

the exception of viscosity. Almost all women ( $\mathrm{N}=31,91.2 \%)$ said if they needed HIV protection they would use a microbicide.

A majority of the men (60\%) similarly expressed approval of their partner's use of the gel if FDA approved. They were motivated by its potential protective qualities $(\mathrm{r}=0.980, \mathrm{p}<0.001)$ against HIV. They also agreed a microbicide would be more acceptable if it had dual protection attributes protecting their partners against HIV, STIs, and pregnancy. It is important to note that the evaluation of responses by male sexual partners likely varies depending on female product assignment. Overall, the likelihood of future gel use was negatively correlated with the product decreasing sexual pleasure. Therefore, future marketing of microbicides should highlight their lubricating properties for sexual enhancement [22-24].

This study offers some unique insights on microbicides from our ethnically diverse participants. Although $100 \%$ of black/African American women $(n=9)$ said they would use the gel if it was approved as a safe and effective strategy, only $40 \%$ of Caucasian women $(\mathrm{N}=4)$ said they would be likely to use the gel. Similarly, most of the black/ African American women $(\mathrm{N}=13,87 \%)$ agreed that it was "very important" to develop a dual protection product that could protect them against HIV, STIs, and pregnancy compared to $44 \%$ of Caucasians $(\mathrm{N}=8)$. In addition, $80 \%$ of black/African American women indicated a preference for the product's added protection against HIV compared to $50 \%$ of white women enrolled. Given the disproportionate impact of HIV/AIDS among minority women in the United States, and especially in the South, these results may be reflective of HIV risk perceptions between ethnic groups and the need for more female-controlled protective options among minority women.

Qualitative data revealed low approval of some of the UC781 product properties. The gel was noticeable for some of the women during sex. It hindered sexual spontaneity and limited certain sexual acts. Some of these product attribute concerns have been identified among other populations [18-20]. Study participants expressed support for product reformulation to avoid leakage, messiness, unpleasant scent, and insertion timing. They recommended a thicker, clear, odorless gel as an alternative. Although the women were familiar with the shape and form of the applicator, the participants indicated a need to reduce the number of gel insertion times.

Women articulated that these negative product attributes would thwart covert use of the product while in stable sexual partnerships. A reformulated product, or a microbicidal vaginal ring, foam, or suppository, would provide women with more options for adopting safer sex practices with their partners.

\section{Limitations}

Limitations include the use of a small sample of women $(\mathrm{N}=34)$ and male partners $(\mathrm{N}=10)$ who met the specified criteria for participation and were willing to join this Phase I study. Although product adherence within the trial was good likely due to the short study duration $(<2$ months), it is uncertain if the controlled conditions of the study impacted acceptability. Additionally, some caution must be taken with interpretation of statistical tests given small sample sizes, particularly group sizes that are $<10$ for the chi-square tests. Finally, the potential for participation bias is noted, as it is possible that the women interviewed for this study may hold strong opinions about microbicides and medical research. As study participants, they may have a greater predisposition to HIV/AIDS health seeking behavior than other women. Opinions and concerns expressed by this sample of respondents may not be reflective of other women and men from diverse ethnic and racial backgrounds. Finally, because interviews and focus groups were conducted by our study team's qualitative researcher, it is possible that participants offered more socially desirable responses than they would have under other conditions.

\section{Conclusions}


Citation: Frew P, Parker K, Horton T, Hixson B, Flowers L, et al. (2012) Assessment of a Microbicide Candidate among a Diverse Cohort of Urban Southern US Women and their Male Sexual Partners. J AIDS Clinic Res S4:004. doi:10.4172/2155-6113.S4-004

Southern minority women, particularly black/African Americans, a rapidly growing group for HIV infection in the United States, would benefit from the development of a safe and effective form of preexposure prophylaxis for HIV infection. Findings from this study provide insight into barriers and motivators for diverse women to accept and recommend a potential HIV microbicide. While many of the women expressed reluctance to take or recommend the existing UC781 product or placebo as currently formulated, there was general agreement that development of an improved HIV/AIDS microbicide was an important endeavor and an improved product would be acceptable to women in the future.

\section{Acknowledgements}

The authors wish to thank the study volunteers for their participation and support of this study. The authors also acknowledge the support by the United State Agency for International Development (USAID) for CONRAD personne involved in the study. This paper does not necessarily reflect the views of USAID.

\section{Sources of Support}

Study support was provided by the Centers for Disease Control and Prevention (200-2003-029494), Emory Center for AIDS Research (P30 Al050409), the Emory HIVIAIDS Clinical Trials Unit (U01 AI069418). Its contents are solely the responsibility of the authors and do not necessarily represent the official views of the United States Centers for Disease Control and Prevention (CDC).

\section{Related Presentations}

This study was orally presented by the first author at the CDC National HIV Prevention Conference in Atlanta, Georgia on August 25, 2009.

\section{References}

1. Centers for Disease Control and Prevention (2007) Racial/Ethnic Disparities in Diagnoses of HIVIAIDS-33 States, 2001-2005. Morbidity and Mortality Weekly. 56: 189-193.

2. Centers for Disease Control and Prevention (2008) HIVIAIDS Surveillance Supplemental Report: Cases of HIV infection and AIDS in the United States and dependent areas, by race/ethnicity, 2002-2006

3. Centers for Disease Control and Prevention (2008) CDC HIVIAIDS Fact Sheet: HIVIAIDS among Women.

4. Wingood G, DiClemente R (2002) The theory of gender and power: A social structural theory for guiding public health interventions. In: DiClemente $\mathrm{R}$, Crosby R, Kegler M, (eds). Emerging theories in public health practice and research. San Francisco, CA: Jossey-Bass Wiley 313-346.

5. Aral S, Wasserheit J (1995) Interactions among HIV, other sexually transmitted diseases, socioeconomic status, and poverty in women. In: O'Leary A, Jemmott L, (eds), Women at Risk: Issues in the Primary Prevention of AIDS. New York: Plenum Press 13-41.

6. Jemmott L, Catan V, Nyamathi A, Anastasia J (1995) African American women and HIV-risk-reduction issues. In: O'Leary A, Jemmott L, (eds). Women at Risk: Issues in the Primary Prevention of AIDS. New York, NY: Plenum Press 131158

7. O'Leary A, Wingood G (2000) Interventions for sexually active heterosexual women. In: Peterson J, DiClemente R, eds. Handbook of HIV Prevention: Kluwer/Plenum Press 179-200.

8. Poynten M, Brown JM, Sovero M, Millwood IY, Kaldor JM (2008) Microbicide safety and effectiveness: An overview of recent clinical trials. Curr Opin HIV AIDS 3: $574-580$

9. Cutler B, Justman $\mathrm{J}$ (2008) Vaginal microbicides and the prevention of HIV transmission. Lancet Infect Dis 8: 685-697.

10. Abdool Karim Q, Abdool Karim SS, Frohlich JA, Grobler AC, Baxter C, et al.
(2010) Effectiveness and safety of tenofovir gel, an antiretroviral microbicide, for the prevention of HIV infection in women. Science 329: 1168-1174

11. Cates W Jr (2010) After CAPRISA 004: time to re-evaluate the HIV lexicon. Lancet 376: 495-496.

12. Kiser PF, Mahalingam A, Fabian J, Smith E, Damain F, et al. Design of tenofovirUC781 combination microbicide vaginal gels. J Pharm Sci 101: 1852-1864.

13. Schwartz JL, Kovalevsky G, Lai JJ, Ballagh SA, McCormick T, et al. (2008) A randomized six day safety study of an antiretroviral microbicide candidate UC-781, a non-nucleoside reverse transcriptase inhibitor. Sex Transm Dis 35: 414-419.

14. Schwartz JL, Ballagh SA, Kwok C, Mauck CK, Weiner DH, et al. (2007) Fourteen-day safety and acceptability study of the universal placebo gel. Contraception 75: 136-141.

15. MacQueen K, McLellan E, Kay K, Milstein B (1998) Codebook development for team-based qualitative analysis. Cultural Anthropology Methods 10: 31-36.

16. Glaser GB, Strauss AL (1967) The discovery of grounded theory: Strategies for qualitative research. Chicago: Aldine.

17. Katzen LL, Fernández-Romero JA, Sarna A, Murugavel KG, Gawareck D, et al. (2011) Validation of a dye stain assay for vaginally inserted hydroxyethylcellulose-filled microbicide applicators. Sex Transm Dis 38: 10501055.

18. Whitehead SJ, Kilmarx PH, Blanchard K, Manopaiboon C, Chaikummao S, et al. (2006) Acceptability of Carraguard vaginal gel use among Thai couples. AIDS 20: 2141-2148.

19. Weeks MR, Mosack KE, Abbott M, Sylla LN, Valdes B, et al. (2004) Microbicide acceptability among high-risk urban U.S. women: Experiences and perceptions of sexually transmitted HIV prevention. Sex Transm Dis 31: 682-690.

20. Rosen RK, Morrow KM, Carballo-Diéguez A, Mantell JE, Hoffman S, et al (2008) Acceptability of Tenovovir gel as a vaginal microbicide among women in a Phase I trial: A mixed methods study. J Womens Health (Larchmt) 17: 383-392.

21. Morrow K, Rosen R, Richter L, Emans A, Forbes A, et al. (2003) The Acceptability of an Investigational Vaginal Microbicide, PRO $2000 \mathrm{Gel}$, among Women in a Phase I Clinical Trial. Journal of Womens Health (Larchmt) 12 655-666.

22. Holt BY, Morwitz VG, Ngo L, Harrison PF, Whaley KJ, et al. (2006) Microbicide preference among young women in California. $\mathrm{J}$ Womens Health (Larchmt) 15: 281-294.

23. Coggins C, Blanchard K, Friedland B (2000) Men's attitudes twoards a potential vaginal microbicide in Zimbabwe, Mexico, and the USA. Reprod Health Matters 8: 132-141.

24. Severy LJ, Tolley E, Woodsong C, Guest G (2005) A framework for examining the sustained acceptability of microbicides. AIDS Behav 9: 121-131.

25. Grady WR, Klepinger DH, Nelson-Wally A (1999) Contraceptive characteristics: The perceptions and priorities of men and women. Fam Plann Perspect 31: 168-175.

This article was originally published in a special issue, Epidemiology and Prevention: HIV handled by Editor(s). Dr. Han-zhu Qian, Vanderbilt University School of Medicine, USA 